\title{
IDENTIFICAÇÃO DE OVINOS E CAPRINOS RESISTENTES E SUSCEPTÍVEIS AOS HELMINTOS GASTRINTESTINAIS
}

\section{Identification of Sheep and Goats Resistant or Susceptible to Gastrointestinal Parasites}

\author{
Cristina Santos Sotomaior \\ Médica Veterinária, Dr. ${ }^{a}$, Prof. ${ }^{a}$ da PUCPR, São José dos Pinhais - PR. e-mail: cssoto@onda.com.br \\ Lorena Moreira De Carli \\ Médica Veterinária, Curitiba - PR. e-mail: loredecarli@gmail.com \\ Leonardo Tangleica \\ Acadêmico de Medicina Veterinária, Curitiba - PR. e-mail: tangleica@hotmail.com \\ Bettina Karla Kaiber \\ Acadêmica de Medicina Veterinária, Curitiba - PR. e-mail: bkaiber@hotmail.com \\ Felipe Pohl de Souza \\ Médico Veterinário, M. Sc., Prof. da PUCPR, São José dos Pinhais - PR. e-mail: f.pohl@globo.com
}

\section{Resumo}

O controle da verminose gastrintestinal de pequenos ruminantes representa grande desafio no manejo sanitário destas espécies. $\mathrm{O}$ aumento de casos de resistência dos parasitos aos diferentes princípios ativos tem gerado uma demanda por alternativas de controle não-químico, como a seleção de animais que são geneticamente resistentes aos parasitos. Com o objetivo de identificar animais resistentes e susceptíveis aos helmintos gastrintestinais, foram realizados dois experimentos, estudando 70 ovelhas e 28 caprinos. A contagem de ovos por gramas de fezes (OPG), valores de hematócrito (Ht), contagem de eosinófilos sanguíneos e classificação pelo método FAMACHA ${ }^{\circ}$ foram utilizados na análise de Cluster para esta identificação. No primeiro experimento, 11 ovelhas $(28,9 \%)$ foram classificadas como resistentes e $11(28,9 \%)$ como susceptíveis. Após a classificação, o rebanho foi acompanhado e as médias dos animais em período de lactação, para o grupo de resistentes e susceptíveis, foram de 435,2 e 3924,0 OPG; e de 1,5 e 1,9 para o FAMACHA ${ }^{\odot}$, respectivamente. Já para as ovelhas fora do período de lactação, as médias foram de 80,0 e 354,4 OPG para resistentes e susceptíveis, respectivamente, e de 1,3 para o FAMACHA $^{\circ}$, para ambos os grupos. No segundo experimento, em 28 caprinos, usando dados do FAMACHA $^{\circ}$, foi possível classificar como susceptíveis 10,7\% dos animais e 17,85\% como resistentes. Quando foram utilizados somente os dados de OPG, também houve separação em grupos. Porém, o resultado obtido pelo FAMACHA ${ }^{\odot}$ é o mesmo do OPG somente quando se tratam dos animais susceptíveis. O FAMACHA ${ }^{\circ}$ identifica animais resilientes (capazes de suportar os efeitos da infecção parasitária) e não necessariamente resistentes (capazes de evitar o estabelecimento da infecção). O método FAMACHA ${ }^{\odot}$ pode ser útil na classificação de animais em susceptíveis e resistentes/resilientes aos helmintos gastrintestinais, desde que utilizado a longo prazo e em rebanhos com maior grau de infecção parasitária.

Palavras-chave: Pequenos ruminantes; Verminose gastrintestinal; Seleção de animais resistentes. 


\begin{abstract}
The effective parasite control in small ruminant production is a great challenge. The increase of anthelmintic resistance has generated a demand for non chemical alternatives of control, as the selection of animals genetically resistant to parasites. With the goal to identify animals resistant and susceptible to gastrointestinal helminths, two trials were carried out, evaluating 70 sheep and 28 goats. Faecal egg count (FEC), hematocrit value, blood eosinophils count and FAMACHA ${ }^{\odot}$ classification where used in the Cluster analysis for the identification. In the first trial, 11 sheep (28.9\%) were classified as resistant and 11 (28.9\%) as susceptible. After the classification, the average of FEC of the lactating ewes, for the group of resistant and susceptible, were 435.2 and 3924.0 EPG respectively, and 1.5 and 1.9 for FAMACHA $A^{\odot}$ classification. For the non lactating ewes, the mean FEC were 80.0 and 354.4 for the resistant and susceptible, respectively, and 1.3 for the FAMACHA ${ }^{\odot}$ for both groups. In the second trial, with 28 goats, using the FAMACHA ${ }^{\odot}$ data in the Cluster analysis, it was possible to classify as susceptible $10.7 \%$ of the animals and $17.85 \%$ as resistant. When the EPG data was used in the analysis, it was also possible the classification. However, the result for the FAMACHA ${ }^{\odot}$ classification is the same for EPG classification only for the susceptible ones. The FAMACHA ${ }^{\odot}$ method identifies resilient animals (capable to support the effect of the parasitic infection) and not necessarily resistant (capable to prevent the establishment of the infection). Thus, the FAMACHA ${ }^{\odot}$ method can be useful in the classification of animals as resistant/resilient and susceptible to gastrointestinal helminths, if it is used for long periods and in high parasitic infections.
\end{abstract}

Keywords: Small ruminant; Gastrointestinal parasite; Selection of resistant animals.

\title{
INTRODUÇÃO
}

No Paraná, as infecções por nematódeos gastrintestinais têm se constituído em importante entrave à expansão da ovinocultura e caprinocultura de corte, destacando-se o Haemonchus contortus como principal parasita. A elevada prevalência associada à grande patogenicidade faz deste parasito o mais nocivo no Estado do Paraná e em diferentes regiões do território nacional (AMARANTE et al., 1992; VIEIRA; CAVALCANTE, 1999; RAMOS et al., 2002). A ovinocultura paranaense está baseada num sistema intensivo de criação, caracterizada por pastagens com altas lotações e, conseqüentemente, grande contaminação de larvas. O uso indiscriminado de anti-helmínticos, como única forma de controle da verminose gastrintestinal, resultou em um problema gravíssimo de resistência nos rebanhos paranaenses (SOCCOL et al., 1996; THOMAZ-SOCCOL et al., 2004). Como a caprinocultura, especialmente para a produção de carne, vem adotando muitas técnicas utilizadas na ovinocultura, em especial os métodos de controle de verminose baseado somente na utilização de anti-helmínticos, também já há relatos de casos de resistência a diferentes princípios ativos em caprinos (SOTOMAIOR; MILCZEWSKI; SCHWARTZ, 2002).

O vermífugo é um recurso necessário, mas não renovável, à medida que a resistência antihelmíntica vem avançando progressivamente sobre os mais modernos grupos químicos disponíveis (NARI, EDDI, 2002). Em ovinos e caprinos, há relatos de resistência a todos os grupos de antihelmínticos: benzimidazóis, lactonas macrocíclicas, imidazotiazóis e salicilanidas (SANGSTER, 1999; THOMAZ-SOCCOL et al., 2004; COLES et al., 2006). A disponibilidade futura de novos antiparasitários está comprometida por este progressivo aumento dos casos de resistência e pelos crescentes custos de pesquisa e desenvolvimento de novas drogas (COLES et al., 2006). Portanto, é urgente que esforços máximos sejam efetuados para desenvolver, validar e utilizar sistemas de Controle Integrado de Parasitos (CIP), para se tentar diminuir os efeitos produzidos pela resistência.

Uma ferramenta para o CIP é o método FAMACHA ${ }^{\circ}$, desenvolvido por pesquisadores sulafricanos para facilitar a identificação clínica de ovinos parasitados por Haemonchus spp. O método FAMACHA $^{\odot}$ consiste em uma avaliação clínica da anemia causada pelo Haemonchus spp., pela comparação 
da coloração da conjuntiva com um padrão preestabelecido, utilizando um cartão. Nesse cartão estão presentes 5 categorias, variando da 1 (coloração vermelho brilhante) até a 5 (coloração pálida, quase branca). Essa divisão representa diferentes médias de valores de hematócritos, sendo 35, 25, 20, 15 e 10 respectivamente para os grupos de 1 a 5 (BATH et al., 2001; VAN WYK, 2002). Baseado nesta comparação, seriam desverminados somente os animais que apresentassem coloração de mucosa compatível com os grupos 4 e 5 e, em alguns casos, com o grupo 3.

Nos rebanhos ovinos, observa-se grande diferença individual na capacidade de resistir ao desafio parasitário. Enquanto a maioria dos animais do rebanho possui contagens de ovos nas fezes (OPG) baixas ou não apresentam sinais clínicos de parasitismo, outros indivíduos apresentam-se anêmicos e altamente parasitados com grande freqüência (SOTOMAIOR, 1997). Uma prática comum é a de se recomendar a desverminação de todos os animais do lote ou da propriedade em função desta pequena porcentagem de animais com alta carga parasitária, utilizando-se a droga sem necessidade na maior parte do lote e aumentando ainda mais a pressão de seleção sobre a população de helmintos. Com o uso do FAMACHA $^{\odot}$, podem seridentificados somente os animais que necessitam da desverminação, diminuindo o número de parasitas sujeitos à ação da droga. Dessa forma, haverá uma população de refúgio com mais parasitos suscetíveis, retardando o aumento da porcentagem resistente (VAN WYK; BATH, 2002). Em estudos recentes, Sotomaior e Caldas (2004) demonstraram que o método pode ser utilizado também em caprinos, desde que respeitadas algumas regras específicas para esta espécie.

Ainda na busca de alternativas de controle, destaca-se também a seleção de animais geneticamente mais resistentes aos parasitos gastrintestinais (LI; MILLER; FRANKE, 2001; BRICARELLO et al., 2004). Uma vez que a resistência aos parasitos gastrintestinais pode ser considerada como a habilidade do animal em impedir o estabelecimento e/ou subseqüente desenvolvimento da infecção parasitária (ALBERS et al., 1990), a quantificação da carga parasitária seria a forma mais direta de se avaliar a resposta do hospedeiro frente à infecção (KASSAI et al., 1990). Assim, a utilidade de determinado método para a identificação da resistência depende da sua relação com a carga parasitária e de sua repetibilidade (STEAR et al., 1995).

Vários possíveis "marcadores" foram e continuam sendo avaliados como forma de identificar os animais geneticamente resistentes às parasitoses. Dentre os principais, destacam-se: hematócrito (WOOLASTON; BARGER; PIPER, 1990; ALBERS et al. 1990), sistema imunológico (GILL 1991; SRÉTER; KASSAI; TAKÁCS, 1994), eosinófilos sanguíneos (BUDDLE et al., 1992), contagem de ovos nas fezes (WINDON; DINEEN; KELLY, 1980; WOOLASTON, 1992; SRÉTER; KASSAI; TAKÁCS, 1994; SOTOMAIOR, 1997). Esse último tem sido o mais utilizado como critério de seleção (GOOD et al., 2006).

Em um rebanho, a proporção de animais resistentes, susceptíveis ou com resistência intermediária varia em função da raça e da idade dos animais. Têm-se como animais mais susceptíveis a infecções por Haemoncus spp os jovens. À medida que estes vão crescendo e amadurecendo, poderão desenvolver imunidade de tal forma que na idade adulta muitos deles poderão apresentar resistência. A raça também tem influência nestas proporções, como exemplo, a raça Santa Inês mostrou-se mais resistente à infecção por nematódeos gastrintestinais, quando comparada à raça Suffolk (AMARANTE, 2004).

A característica de resistência destes animais aos parasitos é herdável, sendo assim a importância de selecionar os animais menos susceptíveis a estes parasitas, formando um plantel futuro que possua certa tolerância (resiliência) aos parasitos. No caso da resistência, a resposta imunológica limita o estabelecimento do parasito. Já no caso da resiliência, os animais são capazes de conviver com os parasitos com redução mínima de produtividade (ALBERS et al., 1987).

Com a implantação de programas de nutrição e melhoramento genético, é possível que a criação de pequenos ruminantes no Brasil se torne progressivamente menos dependente da utilização de drogas antiparasitárias para o controle das infecções helmínticas. Com isso, a utilização de animais geneticamente resistentes pode permitir a redução na freqüência dos tratamentos anti-helmínticos, que além de propiciar economia para o criador, permite reduzir a velocidade do surgimento de populações de nematódeos resistentes aos anti-helmínticos. 
Portanto, o objetivo deste trabalho é utilizar diferentes parâmetros (contagem de ovos nas fezes, valores de hematócrito, número de eosinófilos circulantes e classificação pelo método FAMACHA ${ }^{9}$ ) na escolha de ovinos e caprinos a serem selecionados como resistentes ou sensíveis aos helmintos gastrintestinais.

\section{MATERIAL E MÉTODOS}

\section{Experimento 1}

O experimento 1 foi desenvolvido no setor de ovinocultura da Fazenda Experimental Gralha Azul (FEGA), da Pontifícia Universidade Católica do Paraná (PUCPR). Foram utilizadas 38 ovelhas, em fase final de gestação e durante o período de lactação, sendo 12 da raça Hampshire Down e 26 mestiças.

Estes animais foram divididos em dois lotes. O primeiro lote, constituído por 23 ovelhas, foi acompanhado de 21 de agosto a 30 de setembro de 2002. O segundo lote, com 15 ovelhas em lactação, foi acompanhado de 10 de outubro até 19 de dezembro de 2002. Alguns animais tiveram que ser desverminados ao longo do experimento. As desverminações foram feitas com moxidectin na dose de 200 $\mathrm{mg} / \mathrm{kg}\left(\right.$ Cydectin $\left.^{\circledR}\right)$ e closantel na dose de $15 \mathrm{mg} / \mathrm{kg}\left(\right.$ Galgosantel $\left.^{\circledR}\right)$.

Durante toda esta etapa do experimento, foram colhidas fezes de todas as ovelhas semanalmente (6 colheitas do primeiro lote e 11 colheitas do segundo lote). A colheita de sangue foi realizada quinzenalmente ( 3 colheitas do primeiro lote e 6 colheitas do segundo lote). Essas amostras eram identificadas e encaminhadas ao laboratório da Unidade Hospitalar para Animais de Fazenda (UHAF) da PUCPR para a realização da contagem de ovos por grama de fezes (OPG), do hematócrito e contagem dos eosinófilos.

Em seguida, foi realizada a seleção dos animais em resistentes e susceptíveis, pela análise de Cluster, utilizando todas as características estudadas.

Das 38 ovelhas selecionadas, 15 ovelhas mestiças e 16 da raça Hampshire Down, totalizando 31 animais, foi realizado o acompanhamento, utilizando valores de OPG e o método FAMACHA ${ }^{\circ}$. Os exames de OPG e a avaliação do método FAMACHA ${ }^{\odot}$ iniciaram-se em agos to até final de setembro 2003 (período de lactação), retornando em fevereiro até o mês de junho de 2004, com as ovelhas em estação de monta, gestação e lactação. Até junho de 2004 foram realizadas 17 avaliações.

\section{Experimento 2}

O segundo experimento consistiu no acompanhamento quinzenal de 28 caprinos da UHAFPUCPR e 32 ovinos de uma propriedade próxima à FEGA, de agosto de 2006 a julho de 2007. Em ambos os rebanhos, os animais eram mestiços de diferentes raças. Durante cada visita, todos os animais eram avaliados pelo método $\mathrm{FAMACHA}^{\odot}$. Além da avaliação do $\mathrm{FAMACHA}^{\odot}$, também eram realizados exames parasitológicos a cada quarenta e cinco dias de todos os animais.

Após as análises dos dados de OPG e FAMACHA ${ }^{\circ}$, fez-se a seleção dos animais em resistentes e susceptíveis, utilizando-se a análise de Cluster.

Para os dois experimentos, as técnicas utilizadas foram as mesmas. As colheitas de fezes eram feitas de cada animal individualmente, com sacos plásticos, diretamente da ampola retal. O número de ovos por grama de fezes (OPG) foi determinado segundo o método de Gordon e Whitlock (1939), sensível para 50 OPG. A cultura e identificação de larvas foram realizadas segundo o método de Roberts e O’Sullivan (1950).

O sangue era colhido da veia jugular externa, em tubos de vacutainer contendo EDTA como anticoagulante. Para a determinação do hematócrito $(\mathrm{Ht})$, utilizaram-se métodos de rotina, segundo Jain (1986). O Ht, dado em porcentagem, foi determinado pelo método do microhematócrito. A contagem do número de eosinófilos sangüíneos foi feita pelo método direto, segundo Lima et al. (1985), utilizando-se a câmara de contagem de Fuchs-Rosenthal e o líquido diluidor de Pilot Modificado (MacFARLANE; CECIL, 1951). 
A avaliação do método FAMACHA ${ }^{\odot}$ foi feita conforme Van Wyk e Bath (2002). A metodologia em cada avaliação consistia na contenção dos animais e posterior comparação da coloração da mucosa com o cartão do método FAMACHA $^{\odot}$. Essas colorações estão divididas em 5 categorias correspondentes a valores específicos de hematócrito (Ht e" 28: grau 1; entre 23-27: grau 2; entre 18 e 22 : grau 3; entre 13-17: grau 4; d" 12: grau 5). Uma vez determinada qual a classificação feita pelo método FAMACHA $^{\circ}$, os dados eram registrados.

\section{Análise estatística}

Para a classificação dos animais em resistentes e susceptíveis nos dois experimentos, utilizouse a análise de Cluster, calculadas com dados de OPG, eosinófilos, hematócrito e classificações de

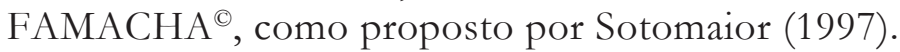

$\mathrm{Na}$ análise de Cluster, as unidades, representadas pelas ovelhas e os caracteres (OPG, hematócrito, número de eosinófilos e classificações de FAMACHA ${ }^{\odot}$ ) foram submetidos a um processo de análise de agrupamento. O agrupamento foi feito com base na similaridade ou na distância. Os resultados são dispostos graficamente em um diagrama em árvore, ou dendograma, que possui uma escala para se observar os níveis de agrupamento. São utilizadas ligações completas e a distância euclidiana. A análise de Cluster por si só somente agrupa os animais. A definição de resistentes ou susceptíveis deve ser feita pela análise bruta dos dados.

Para a comparação das médias dos valores do OPG, Ht, eosinófilos e classificações do FAMACHA $^{\odot}$ dos grupos de animais considerados resistentes ou susceptíveis, utilizou-se o teste nãoparamétrico de U de Mann-Whitney, considerando o valor de $\mathrm{p}<0,05$ como estatisticamente significante.

\section{RESULTADOS}

\section{Experimento 1}

$\mathrm{Na}$ realização dos exames parasitológicos, foi possível a observação de diferentes tipos de ovos e oocistos, como ovos tipo estrongiliformes, ovos de Strongyloides papillosus, Monię̧ia sp e oocistos de Eimeria sp. Para as análises posteriores, foram consideradas as contagens de ovos (OPG) somente de estrongilídeos.

Considerando todas as análises do primeiro e segundo lotes, valores máximos de até 64200 OPG foram observados, enquanto alguns animais mantiveram OPG muito baixo ou nulos durante todas as avaliações.

Com relação à cultura de larvas, ficou evidenciado que a principal larva presente nas amostras foi o Haemonchus contortus (86\%). As demais encontradas foram do gênero Trichostrongylus (13\%) e Cooperia (1\%).

Os valores médios Ht variaram de 13 a 39\% e a contagem de eosinófilos circulantes, de 25 a 3328,10 eosinófilos $/ \mathrm{mm}^{3}$.

Baseado nestes dados de OPG, eosinófilos e hematócritos, foi possível, por meio da análise de Cluster, a separação dos ovinos em resistentes e susceptíveis pelos dendogramas formados (FIGURAS 1; 2). Das 38 ovelhas estudadas, 11 (28,9\%) foram classificadas como resistentes e 11 (28,9\%) como susceptíveis. A análise de Cluster foi realizada com todos os dados (OPG, valores de hematócrito e contagem de eosinófilos), em ambos os lotes, mas analisados separadamente.

$\mathrm{Na}$ análise do primeiro lote, foi observado que as linhas do gráfico estavam muito próximas, dificultando a visualização da posição que alguns animais ocupavam. Isso ocorreu devido à grande distância de 3 animais (HV 122, 263 e HV 05), que puderam ser classificados como susceptíveis de acordo com o posicionamento no diagrama, corroborados pelos altos valores dos seus respectivos exames de OPG. Essa distância ocorre devido a valores muito diferentes dos demais. Para solucionar o problema no dendograma, foi realizada uma segunda análise, excluindo estes animais, já considerados altamente susceptíveis (FIGURA 1). 


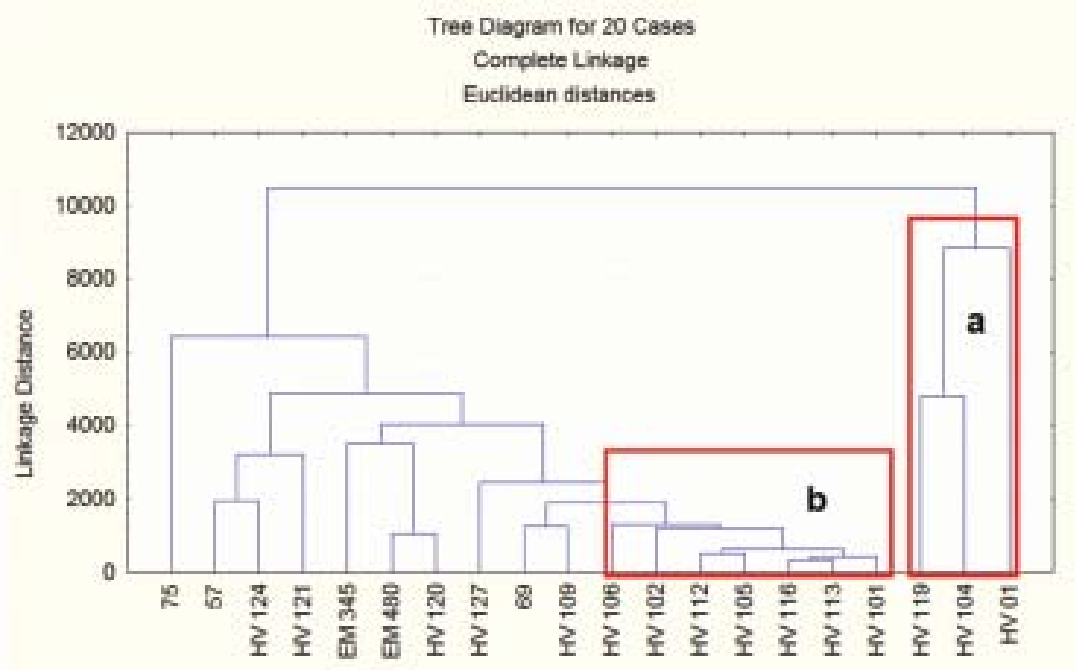

FIGURA 1 - Dendograma com valores de hematócrito, eosinófilos e OPG do $1^{\circ}$ lote sem os animais $\mathrm{HV}$ 122, HV 05 e 263

Figure 1 - Dendogram made with packed cell volume (PCV), eosinophil count and faecal egg count from first group without three animals (HV 122, HV 05 and 263) already considered as susceptible

Na Figura 1, utilizando o padrão hierárquico ascendente, foi possível visualizar e classificar os animais pelos grupos formados "a" - animais susceptíveis e "b" - animais resistentes. A análise conjunta dos dados, principalmente de OPG, auxiliou na delimitação do grupo a.

Com relação ao segundo lote, a montagem do dendograma foi direta, sem necessidade de exclusões (FIGURA 2).

$\mathrm{Na}$ interpretação, foi possível observar os grupos formados “a" e "b", respectivamente animais que são classificados como susceptíveis e resistentes. Essa classificação, assim como no primeiro lote, é corroborada pela comparação com os valores dos exames de OPG.

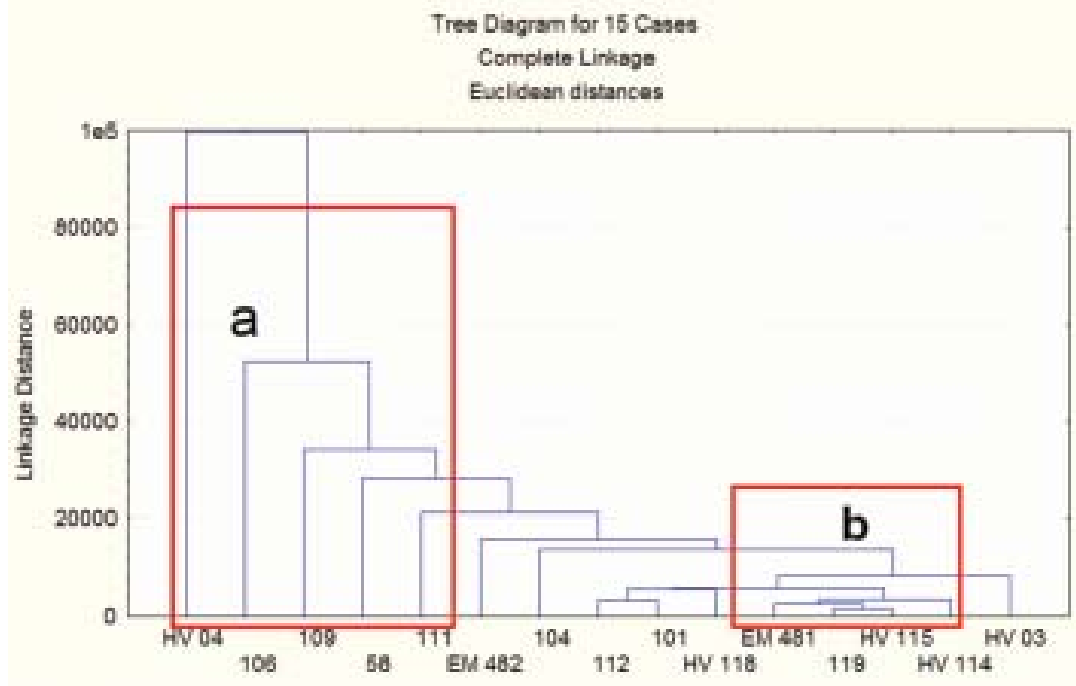

FIGURA 2 - Dendograma com os valores com valores de hematócrito, eosinófilos e OPG do $2^{\circ}$ lote.

Figure 2 - Dendogram made with PCV, eosinophil count and faecal egg count from second group 
Após a classificação, observa-se que no grupo dos animais resistentes, a média de contagem de ovos foi 129,55 OPG, enquanto a média obtida nas susceptíveis foi de 6809,09 OPG. Os valores médios de hematócrito e eosinófilos para as resistentes foram de $29,7 \%$ e $456,18 \mathrm{~mm}^{3}$, respectivamente e para as susceptíveis, 22,83\% e 455,38 $\mathrm{mm}^{3}$ (TABELA 1).

TABELA 1 - Média da contagem de ovos nas fezes (OPG), do hematócrito (\%) e do número de eosinófilos $\left(/ \mathrm{mm}^{3}\right)$ das ovelhas classificadas como resistentes $(\mathrm{R})$ ou susceptíveis $(\mathrm{S})$

Table 1 - Mean faecal egg count, PCV (\%) and eosinophil count $\left(/ \mathrm{mm}^{3}\right)$ from ewes considered resistant $(\mathrm{R})$ or susceptible (S)

\begin{tabular}{lc|c|c|c|c|c}
\hline & \multicolumn{2}{c}{$\mathrm{OPG}^{-}$} & \multicolumn{2}{c}{ Eosinótilos } \\
\cline { 2 - 7 } & $\mathrm{R}$ & $\mathrm{S}$ & $\mathrm{R}$ & $\mathrm{S}$ & $\mathrm{R}$ & $\mathrm{S}$ \\
\hline Ovelhas & $129,5^{* *}$ & $6809,1^{* *}$ & $29,7^{*}$ & $22,83^{*}$ & 456,18 & 455,38 \\
\hline
\end{tabular}

Diterença signitıcatıva ( $p<v, v$ ) entre o grupo resistente (K) e susceptıveı (D).

${ }^{* *}$ Diferença significativa $(\mathrm{p}<0,01)$ entre o grupo resistente $(\mathrm{R})$ e susceptível (S).

Após a classificação, houve a continuidade de acompanhamento com dados de OPG e FAMACHA $^{\odot}$. A média dos valores de OPG de todas as coletas realizadas, do grupo de ovelhas resistentes e susceptíveis, foi comparada. No período de lactação e final de gestação (30/abr), nota-se que a média do grupo das ovelhas susceptíveis é superior às resistentes, sendo esta diferença estatisticamente significativa $(\mathrm{p}<0,05)$ em três avaliações. $\mathrm{Na}$ fase de início de gestação, não é possível evidenciar esta diferença (TABELA 2).

TABELA 2 - Média dos valores de OPG das ovelhas classificadas como resistentes e susceptíveis nas 17 avaliações

Table 2 - Average faecal egg count from ewes considered resistant or susceptible in each evaluation date

\begin{tabular}{|c|c|c|c|}
\hline & \multicolumn{2}{|c|}{ Média OPG } & \multirow{8}{*}{$1^{\circ}$. Periodo de lactaçio } \\
\hline & Resistentes & Susceptiveis & \\
\hline $21 /$ ago & $333,3=$ & 225,0 " & \\
\hline $30 /$ ago & $100,0=$ & $4483,3 \mathrm{~b}$ & \\
\hline $04 /$ set & $400,0=$ & 23833,3 a & \\
\hline $13 /$ set & $100,0^{=}$ & $2625,0^{=}$ & \\
\hline $18 /$ set & $23,3,3=$ & $2900,0=$ & \\
\hline $27 /$ set & $750,0^{=}$ & $3325,0=$ & \\
\hline $03 /$ fev & $0,0=$ & $25,0^{=}$ & \multirow{8}{*}{ Inicio e final de gestaçiò } \\
\hline $20 /$ fev & $37,5=$ & $0,0=$ & \\
\hline $05 / \operatorname{mar}$ & $60,0=$ & $10,0^{2}$ & \\
\hline $18 / \operatorname{mar}$ & $0,0=$ & $100,0=$ & \\
\hline $02 /$ abr & $112,5=$ & $166,7 \mathrm{a}$ & \\
\hline $30 / a b r$ & $270,0=$ & $1825,0^{b}$ & \\
\hline $13 / \mathrm{mai}$ & $50,0^{2}$ & $350,0=$ & \\
\hline $04 /$ jun & $440,0=$ & 662,5 a & \\
\hline $11 /$ jun & $1020,0=$ & $1860,0^{=}$ & \multirow[t]{5}{*}{ 2. Periodo de lactação } \\
\hline 18 /jun & $450,0^{=}$ & $1390,0^{b}$ & \\
\hline 25/jun & $910,0=$ & $1510,0^{=}$ & \\
\hline Média lactaçào & 435,2 & 3924,0 & \\
\hline Média periodo seco & 80,0 & 354,4 & \\
\hline
\end{tabular}

Obs.: Letras diferentes na mesma linha significam diferença estatística $(\mathrm{p}<0,05)$. 
Quanto às médias dos valores de $\mathrm{FAMACHA}^{\circledR}$, não foram observadas diferenças significativas entre o grupo das ovelhas resistentes e susceptíveis em nenhuma das avaliações (TABELA 3 e FIGURA 3).

TABELA 3 - Média dos valores de FAMACHA $^{\odot}$ das ovelhas classificadas como resistentes e susceptíveis nas 17 avaliações

Table 3 - Average FAMACH $A^{\odot}$ values from ewes considered resistant or susceptible in each evaluation date

\begin{tabular}{|c|c|c|c|}
\hline \multicolumn{3}{|c|}{ Média FAMACHA } & \multirow{7}{*}{$1^{\circ}$. Período de lactação } \\
\hline 21/ago & $1,8=$ & $2,3=$ & \\
\hline 30/ago & $2,0^{\text {a }}$ & $2,0^{\text {n }}$ & \\
\hline $04 /$ set & $1,3=$ & $2,0=$ & \\
\hline $13 /$ set & $1,5^{\text {a }}$ & $2,0=$ & \\
\hline $18 /$ set & 1,8 * & 2,7 a & \\
\hline $27 /$ set & 1,6 " & $2,0^{\circ}$ & \\
\hline $03 /$ fev & 1,2 " & 1,3 a & \\
\hline $20 /$ fev & 1,3 a & $1,3=$ & \\
\hline $05 / \mathrm{mar}$ & 1,4 * & 1,2 * & Início e final de gestação \\
\hline $18 / \mathrm{mar}$ & 1,3 a & 1,0 a & \\
\hline $02 / \mathrm{abr}$ & 1,3 a & 1,0 a & \\
\hline $30 / \mathrm{abr}$ & 1,2 * & $1,5^{\text {a }}$ & \\
\hline $13 /$ mai & $1,2^{\text {s }}$ & $1,5^{\text {* }}$ & \\
\hline $04 /$ jun & 1,2 * & 1,6 " & \\
\hline 11/jun & $1,4^{\text {in }}$ & $1,6^{\text {a }}$ & $2^{\circ}$. Período de lactação \\
\hline $18 /$ jun & 1,2 a & $1,6=$ & \\
\hline $25 /$ jun & $1,2^{\text {a }}$ & $1,4^{\text {* }}$ & \\
\hline Média lactação & 1,5 & 1,9 & \\
\hline Média periodo seco & 1,3 & 1,2 & \\
\hline
\end{tabular}

É possível observar que próximo ao período de lactação e neste propriamente dito, a tendência é o aumento das médias de OPG e FAMACHA ${ }^{\circ}$. 


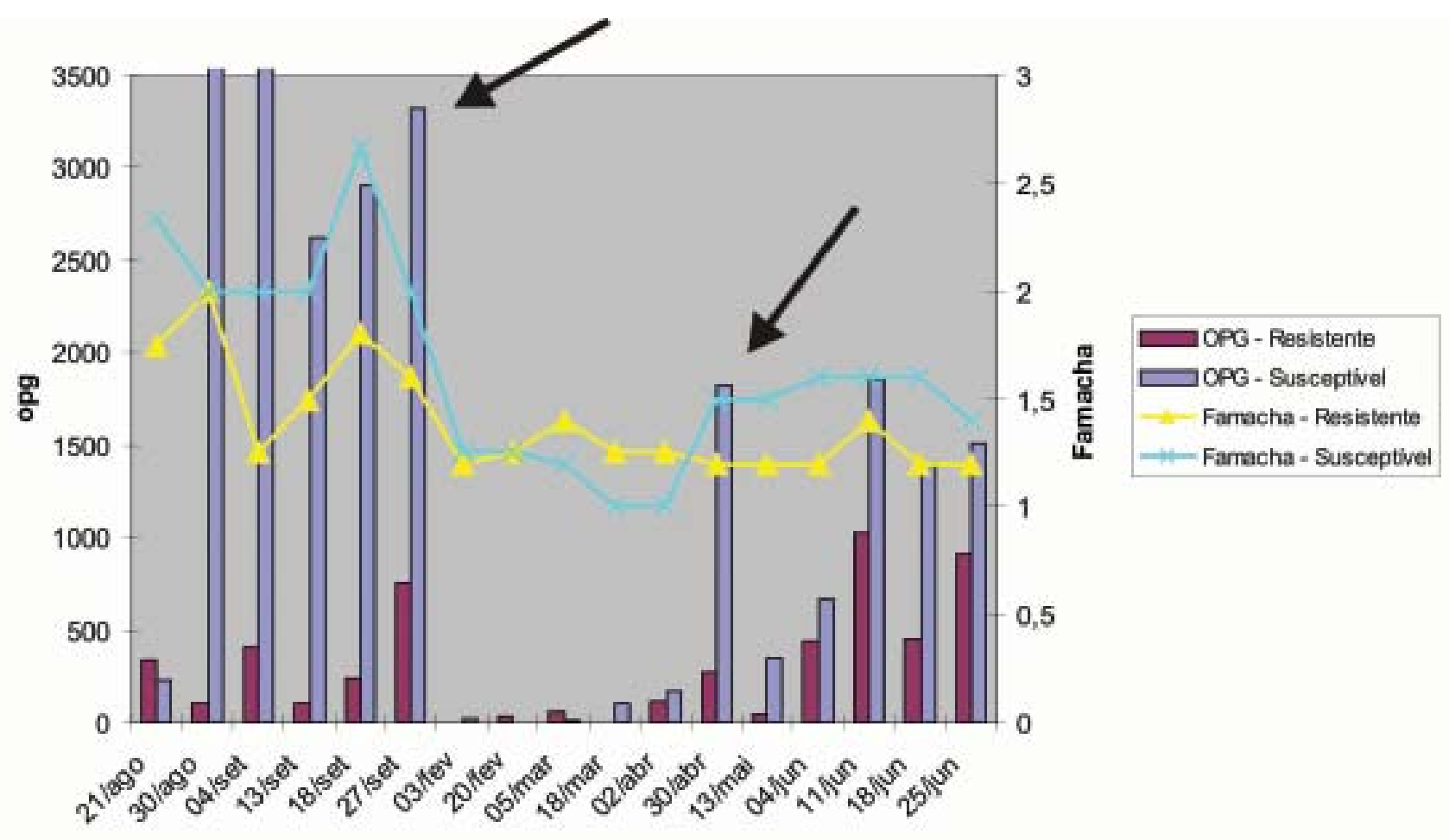

FIGURA 3 - Valores de FAMACHA ${ }^{\odot}$ e OPG dos animais susceptíveis e resistentes em todo o período do experimento, as setas indicam desverminação

Figure 3 - FAMACHA ${ }^{\odot}$ and faecal egg count values from ewes considered resistant or susceptible, arrows indicate anthelmintic treatment

\section{Experimento 2}

Considerando as avaliações pelo método $\mathrm{Famacha}^{\circledR}$ nas duas propriedades, foi possível totalizar 1128 análises individuais, sendo 16 avaliações quinzenais dos 32 ovinos e 22 avaliações dos 28 caprinos.

Analisando as 1128 avaliações do método FAMACHA ${ }^{\circ}$ de acordo com classificações do método (classificação em graus 1, 2, 3, 4 ou 5), observa-se que na propriedade de ovinos há uma predominância de animais classificados como grau 1 (média de 81\%) e nenhuma classificação como 4, indicando que os animais estavam em boas condições sanitárias, com baixos índices de verminose (TABELA 4). Na propriedade de caprinos, a predominância é de animais com grau 2, havendo em média $14,87 \%$ de classificações grau 3 e 3,33\% de grau 4 (TABELA 5). Em nenhuma avaliação, durante todo período experimental, foram encontrados animais classificados como grau 5. 
TABELA 4 - Classificação nos graus 1, 2, 3, 4 e 5 do método FAMACHA ${ }^{\odot}$ na propriedade de ovinos, com 16 avaliações de 32 animais

Table 4 - FAMACHA ${ }^{\circ}$ classification as 1, 2, 3, 4 and 5 in sheep farm, with 16 evaluations of 32 animals

\begin{tabular}{l|r|r|r|r|r}
\hline $\mathrm{N}^{\circ}$ da & $\begin{array}{r}\mathrm{F} 1 \\
\%\end{array}$ & $\begin{array}{r}\mathrm{F} 2 \\
\%\end{array}$ & $\begin{array}{r}\mathrm{F} 3 \\
\%\end{array}$ & $\begin{array}{r}\mathrm{F} 4 \\
\%\end{array}$ & $\begin{array}{r}\mathrm{F5} \\
\%\end{array}$ \\
\hline 1 & 100,0 & 0,0 & 0,0 & 0,0 & 0,0 \\
2 & 96,8 & 3,2 & 0,0 & 0,0 & 0,0 \\
3 & 78,1 & 21,9 & 0,0 & 0,0 & 0,0 \\
4 & 87,5 & 12,5 & 0,0 & 0,0 & 0,0 \\
5 & 87,5 & 12,5 & 0,0 & 0,0 & 0,0 \\
6 & 87,5 & 9,4 & 3,1 & 0,0 & 0,0 \\
7 & 84,4 & 15,6 & 0,0 & 0,0 & 0,0 \\
8 & 68,8 & 31,3 & 0,0 & 0,0 & 0,0 \\
9 & 96,9 & 3,1 & 0,0 & 0,0 & 0,0 \\
10 & 67,7 & 25,8 & 6,5 & 0,0 & 0,0 \\
11 & 61,3 & 29,0 & 9,7 & 0,0 & 0,0 \\
12 & 66,7 & 29,6 & 3,7 & 0,0 & 0,0 \\
13 & 88,9 & 11,1 & 0,0 & 0,0 & 0,0 \\
14 & 78,9 & 21,1 & 0,0 & 0,0 & 0,0 \\
15 & 84,2 & 15,8 & 0,0 & 0,0 & 0,0 \\
16 & 68,4 & 21,1 & 10,5 & 0,0 & 0,0 \\
\hline Média & 81,5 & 16,4 & 2,1 & 0,0 & 0,0 \\
\hline
\end{tabular}

Nos exames coproparasitológ1cos, toram observados ovos de tipo estrong1litorme, Nematodirus sp, Strongyloides papillosus, Moniezia sp e oocistos de Eimeria sp. Novamente, foi considerada apenas a contagem de ovos (OPG) de estrongilídeos.

A média de OPG encontrada foi calculada segundo a categoria de $\mathrm{FAMACHA}^{\odot}$ que o animal se encontrava, como demonstrado na Tabela 6. Nota-se o aumento da média do OPG em cada categoria de FAMACHA ${ }^{\circ}$.

TABELA 5 - Classificação nos graus 1, 2, 3, 4 e 5 do método FAMACHA ${ }^{\odot}$ na propriedade de caprinos, com 22 avaliações de 28 animais

Table 5 - FAMACH $A^{\odot}$ classification as 1, 2, 3, 4 and 5 in goat farm, with 22 evaluations of 28 animals

\begin{tabular}{l|c|c|c|c|c}
\hline $\begin{array}{l}\mathrm{N}^{\circ} \text { da } \\
\text { avaliação }\end{array}$ & $\begin{array}{c}\mathrm{F} 1 \\
\%\end{array}$ & $\begin{array}{c}\mathrm{F} 2 \\
\%\end{array}$ & $\begin{array}{c}\mathrm{F} 3 \\
\%\end{array}$ & $\begin{array}{c}\mathrm{F} 4 \\
\%\end{array}$ & $\begin{array}{c}\text { F5 } \\
\%\end{array}$ \\
\hline 1 & 72,22 & 27,78 & 0,00 & 0,00 & 0,00 \\
2 & 55,56 & 38,89 & 5,56 & 0,00 & 0,00 \\
3 & 58,82 & 35,29 & 5,88 & 0,00 & 0,00 \\
4 & 29,17 & 50,00 & 20,83 & 0,00 & 0,00 \\
5 & 16,67 & 79,17 & 4,17 & 0,00 & 0,00 \\
6 & 27,27 & 54,55 & 18,18 & 0,00 & 0,00 \\
7 & 48,15 & 37,04 & 14,81 & 0,00 & 0,00 \\
8 & 47,83 & 39,13 & 8,70 & 4,35 & 0,00 \\
9 & 21,43 & 57,14 & 14,29 & 7,14 & 0,00 \\
10 & 46,15 & 30,77 & 19,23 & 3,85 & 0,00 \\
11 & 18,52 & 59,26 & 22,22 & 0,00 & 0,00 \\
12 & 14,81 & 29,63 & 37,04 & 18,52 & 0,00 \\
13 & 39,29 & 53,57 & 7,14 & 0,00 & 0,00 \\
14 & 10,71 & 75,00 & 14,29 & 0,00 & 0,00 \\
15 & 14,29 & 67,86 & 17,86 & 0,00 & 0,00 \\
16 & 62,96 & 29,63 & 7,41 & 0,00 & 0,00 \\
17 & 33,33 & 51,85 & 14,81 & 0,00 & 0,00 \\
18 & 37,04 & 48,15 & 7,41 & 7,41 & 0,00 \\
19 & 39,29 & 50,00 & 10,71 & 0,00 & 0,00 \\
20 & 40,00 & 24,00 & 28,00 & 8,00 & 0,00 \\
21 & 54,17 & 33,33 & 12,50 & 0,00 & 0,00 \\
22 & 0,00 & 40,00 & 36,00 & 24,00 & 0,00 \\
\hline Média & 35,80 & 46,00 & 14,87 & 3,33 & 0,00 \\
\hline
\end{tabular}

Rev. Acad., Curitiba, v. 5, n. 4, p. 397-412, out./dez. 2007 
TABELA 6 - Média de OPG de estrongilídeos de cada uma das categorias observadas do método FAMACHA ${ }^{\odot}$ nas propriedades de ovinos e caprinos Table 6 - Average faecal egg count from each $\mathrm{F} A M A C H A^{\odot}$ category in sheep and goat farm

\begin{tabular}{l|c|c}
\hline FAMACHA $^{\circ}$ & $\begin{array}{c}\text { Média de OPG } \\
\text { Propriedade OVI }\end{array}$ & $\begin{array}{c}\text { Média de OPG } \\
\text { Propriedade CAP }\end{array}$ \\
\hline 1 & 160,9 & 860,0 \\
2 & 624,5 & 1250,0 \\
3 & 1065,1 & 3600,0 \\
4 & 1650,0 & 4000,0 \\
\hline
\end{tabular}

Obs.: Em nenhuma avaliação foram encontrados animais classificados como FAMACHA $^{\circ} 5$.

A classificação dos animais das propriedades OVI e CAP foi realizada utilizando a análise de Cluster de duas formas: somente com os dados de FAMACHA $^{\odot}$ e somente com os dados de OPG.

$\mathrm{Na}$ propriedade de caprinos, a análise dos dados somente de FAMACHA $^{\odot}$ permitiu a separação de vários grupos (FIGURA 4), com distâncias variáveis em si. Analisando-se os dados brutos, identifica-se, dentre os grupos formados, o grupo de animais susceptíveis (letra a) e o grupo de animais resistentes (letra b). Os três animais classificados como susceptíveis representam 10,7\% e os cinco animais resistentes, $17,85 \%$. Os demais poderiam ser considerados como intermediários.

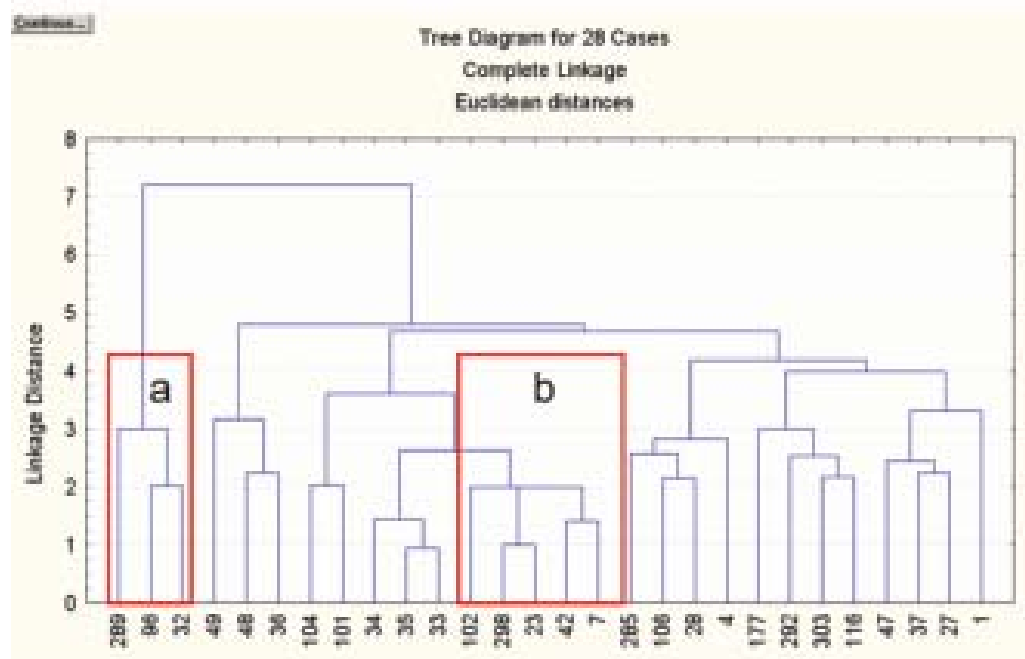

FIGURA 4 - Dendograma com valores somente de classificação pelo método FAMACHA $^{\odot}$ dos 28 caprinos avaliados na propriedade CAP

Figure 4 - Dendogram made with $\mathrm{F} A M A C H A^{\odot}$ values from 28 goats evaluated in farm $C A P$

Quando foram utilizados somente os dados de OPG dos animais da propriedade CAP, também houve uma separação em grupos (FIGURA 5). Porém, quando se comparam os animais agrupados no dendograma formado com os dados do $\mathrm{FAMACHA}^{\odot}$, somente o grupo de animais susceptíveis (letra a) pode ser observado nos dois dendogramas. Os animais agrupados como resistentes pelo FAMACHA ${ }^{\odot}$ estão espalhados em vários grupos neste dendograma do OPG. 


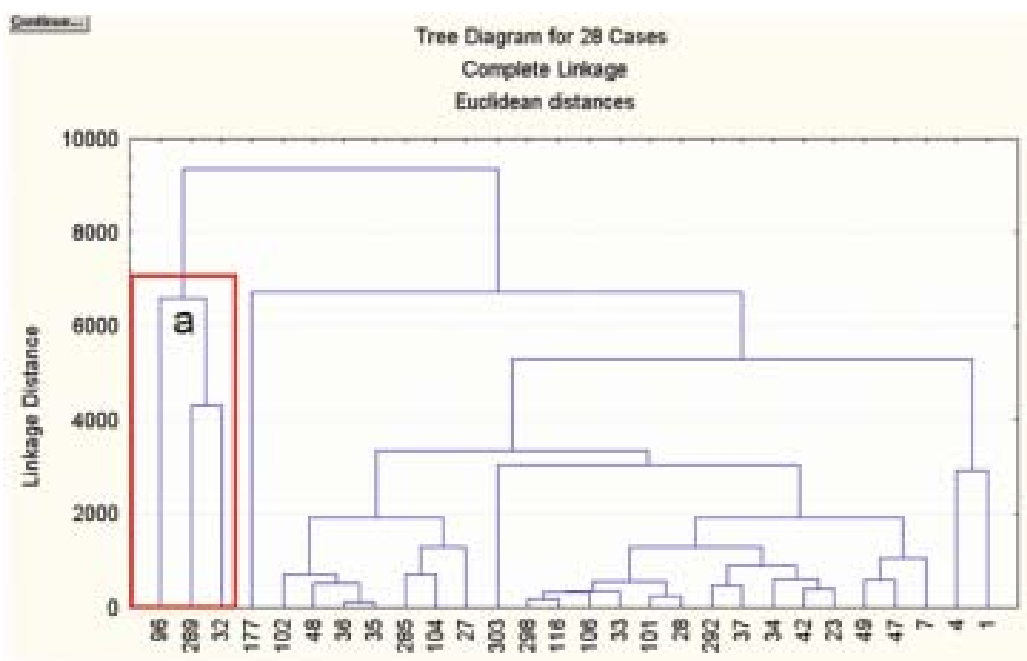

FIGURA 5 - Dendograma com valores somente de OPG dos 28 caprinos avaliados na propriedade CAP

Figure 5 - Dendogram made with faecal egg count values from 28 goats evaluated in farm CAP

Na propriedade OVI, a análise de Cluster, utilizando somente os dados do FAMACHAÓ ou somente os dados de OPG, também não permitiu a separação de grupos que pudessem ser posteriormente classificados como resistentes ou susceptíveis.

\section{DISCUSSÃO}

O controle da verminose gastrintestinal em pequenos ruminantes está passando por uma fase de reavaliação das técnicas utilizadas. Em função dos altos índices de resistências dos parasitos aos diferentes princípios ativos, novas alternativas de controles têm que ser estudadas para se poder diminuir a necessidade de utilização dos anti-helmínticos. O uso de estratégias que não necessitem de desverminações massivas são necessárias na tentativa de retardar o aparecimento da resistência parasitária e/ou para conseguir conciliar a produção ovina intensiva em propriedades que já possuem cepas resistentes. Este é o objetivo do Controle Integrado de Parasitas (CIP).

Certamente a seleção de animais geneticamente resistentes aos helmintos gastrintestinais é uma importante ferramenta deste CIP. A possibilidade de seleção dos mais resistentes, ou mesmo eliminação dos susceptíveis, acarreta uma sensível diminuição da contaminação das pastagens, diminuindo também a necessidade de tratamentos com anti-helmínticos (SOTOMAIOR, 1997).

A metodologia empregada no experimento 1 permitiu separar os animais em resistentes e susceptíveis. As características escolhidas para serem avaliadas (OPG, ht e eosinófilos) estão diretamente relacionadas aos parasitos e como os hospedeiros reagem à sua infecção.

Em tecidos parasitados, a maioria dos componentes do infiltrado celular é o eosinófilo sanguíneo (DORCHIES et al., 1997). Buddle et al. (1992) observaram uma significativa correlação negativa entre eosinofilia sanguínea e OPG, que foi relacionada ao "status" de resistência dos ovinos aos parasitos. Portanto, segundo esses autores, a eosinofilia estaria mais associada com a expressão de resistência aos nematódeos, do que como indicador da presença da infecção, servindo para avaliar a resposta imunológica. Sotomaior (1997) observou diferença estatística significativa quanto ao número de eosinófilos sangüíneos entre o grupo de ovelhas consideradas resistentes e o grupo considerado susceptível, sendo mais elevado nas resistentes. Neste trabalho não foi possível encontrar diferença na média do número de eosinófilos nos grupos resistente e susceptível, talvez por incluir análises em diferentes situações de susceptibilidade dos animais. 
Já os valores de hematócrito refletem o grau de anemia dos animais, sendo importantes para a avaliação de parasitos hematófagos. Neste trabalho, a cultura de larvas evidenciou que a maioria dos parasitos infectantes era o $H$. contortus (86\%), que é hematófago. O H. contortus é o principal nematódeo patogênico em zonas úmidas subtropicais (WALLER, 1997). Isso explica a forte prevalência do parasito no rebanho estudado e confirma a importância do hematócrito para a classificação dos animais. Nos animais estudados foi possível observar diferença estatística nos valores de Ht entre os animais considerados resistentes e susceptíveis (TABELA 1).

Os valores de OPG mostraram claramente a diferença da infecção de um animal para o outro. Enquanto alguns animais nas mesmas condições de manejo e, portanto, com as mesmas chances de contaminação apresentam OPG zero, outros chegam a valores superiores a 64.000. Vários autores mostram diferenças como estas (ALBERS et al., 1987; KASSAI et al., 1990; SRÉTER; KASSAI; TAKÁCS, 1994; SOTOMAIOR, 1997).

Todas estas características podem contribuir para a classificação. Stear et al. (1995) sugerem que a utilização de mais de uma variável, concomitante, poderia fornecer uma identificação mais efetiva dos animais resistentes e susceptíveis. Nesse sentido, buscou-se uma metodologia capaz de permitir a análise dos diferentes parâmetros em conjunto. Assim, utilizou-se a análise de Cluster, com todos os dados do rebanho estudado (OPG, Ht e eoosinófilos). Kassai et al. (1990) sugere que, devido a diferenças de fatores como raça, sexo do hospedeiro, cepa do parasito, nutrição, as características de animais resistentes ou susceptíveis devem ser identificadas para cada rebanho ou sistema de criação.

Após a seleção, o acompanhamento dos animais classificados como resistentes e susceptíveis, quanto à contagem de ovos nas fezes e quanto ao método FAMACHA em diferentes fases (início, final de gestação e lactação), demonstrou diferenças, sendo que em lactação, animais resistentes sempre mantiveram valores de OPG inferiores e valores de FAMACHA ${ }^{\odot}$ superiores aos susceptíveis. Já no período de início de gestação, essa diferença não é evidente, indicando que este não é o período adequado para classificar os animais, pelo baixo grau de infecção parasitária. Sotomaior e Soccol (2001) também observaram que as ovelhas em lactação constituem a categoria mais susceptível às parasitoses gastrintestinais.

As médias dos valores de OPG e FAMACHA ${ }^{\odot}$ dos animais resistentes e susceptíveis são bem distintas. Ao observar o gráfico (FIGURA 3), fica clara esta diferença. Porém, estatisticamente, poucos valores de OPG e nenhum valor de $\mathrm{FAMACHA}^{\odot}$ tiveram resultados diferentes. Isso pode ter ocorrido pelo baixo número de animais utilizados no experimento.

O experimento 2 objetivou introduzir mais uma característica como possível selecionadora. Já existem estudos que demonstram que há uma grande diferença individual entre os animais na capacidade de resistir ou não ao desafio parasitário (WINDON; DINEEN; KELLY, 1980; WOOLASTON, 1992; SRÉTER; KASSAI; TAKÁCS, 1994; SOTOMAIOR, 1997; GOOD et al., 2006).

Ainda que não exista uma correlação direta entre dados de OPG e a classificação pelo método FAMACHA ${ }^{\odot}$, seria normal também haver animais que apresentam somente classificação entre 1 e 2 e aqueles com maior percentagem de valores 3, 4 ou 5. É exatamente esta diferença que norteia o princípio de utilização do método $\mathrm{FAMACHA}^{\odot}$, podendo ser identificados apenas os animais que realmente necessitam ser desverminados (VAN WYK; BATH, 2002).

A análise dos dados dos caprinos mostra que o resultado obtido pelo FAMACHA ${ }^{\odot}$ é o mesmo do obtido pelo OPG, quando se trata dos animais susceptíveis. Porém, no caso dos animais classificados como resistentes, esta semelhança não é observada. Isso pode ser explicado porque o FAMACHA ${ }^{\circ}$ identifica animais resilientes e não necessariamente resistentes. De acordo com Albers et al. (1987), animais "resilientes" são aqueles que têm a capacidade de suportar os efeitos da infecção, uma vez que esses, mesmo com altos valores de OPG, mantêm suas características físicas e hematológicas preservadas. A resistência, por sua vez, seria a habilidade de o animal para resistir à infecção parasitária. Portanto, "resiliência" é a habilidade do animal para manter níveis produtivos aceitáveis apesar da infecção parasitária.

Assim, a classificação conseguida, utilizando somente os dados do FAMACHA $^{\odot}$, pode representar animais tanto resistentes quanto resilientes. Já os dados observados, quando da classificação, utilizando somente os dados de OPG, refletem a classificação de animais resistentes. 
Com a metodologia empregada neste trabalho, foi possível separar os caprinos em resistentes e susceptíveis. Um dos mais importantes aspectos para a utilização do conceito FAMACHA $^{\odot}$ no campo é que este método de avaliação clínica pode fornecer uma real aproximação do estado hematológico dos animais que sofrem de hematofagia pelos parasitos gastrintestinais, especialmente em baixos valores de hematócrito (VAN WYK, 2002).

Nos ovinos, não foi possível uma separação dos animais em resistentes e susceptíveis. A maior homogeneidade dos dados, não havendo grande variação nas classificações do FAMACHA ${ }^{\odot}$ ao longo do tempo, assim como o baixo índice de parasitismo destes animais, pode ter inviabilizado a classificação.

$\mathrm{O}$ método $\mathrm{FAMACHA}^{\odot}$ apresenta como principal benefício a redução no número de tratamentos, pois os animais são avaliados individualmente e somente alguns são desverminados, baixando a pressão da seleção do H. contortus para a resistência aos anti-helmínticos (VAN WYK, 2002). O método $\mathrm{FAMACHA}^{\circ}$, utilizado a longo prazo, pode ser uma ferramenta importante na seleção de animais mais susceptíveis aos helmintos gastrintestinais, permitindo o descarte destes animais e, paulatinamente, aumentado a porcentagem de animais resistentes/resilientes no rebanho, pois apresenta valores de herdabilidade próximos de 0,5 (BISSET et al., 2001). A aplicação em quase todos os sistemas de produção e o fácil aprendizado do método $\mathrm{FAMACHA}^{\odot}$ justificam sua utilização a campo.

\section{CONCLUSÕES}

Foi possível a identificação de pequenos ruminantes resistentes e susceptíveis aos helmintos gastrintestinais.

A utilização de mais de uma variável (OPG, contagem de eosinófilos, hematócrito) auxilia nesta identificação.

Uma vez classificados como resistentes ou susceptíveis aos helmintos gastrintestinais, observa-se diferença significativa entre os dois grupos nos valores de OPG, hematócrito e FAMACHA ${ }^{\odot}$, principalmente nas categorias mais sensíveis à verminose.

O método FAMACHA ${ }^{\odot}$, utilizado a longo prazo, também pode servir com ferramenta nesta identificação de animais resistentes/resilientes aos helmintos gastrintestinais e, principalmente, dos animais susceptíveis.

\section{REFERÊNCIAS}

ALBERS, G. A. A et al. The effect of Haemonchus contortus infection on haematological parameters in young merino sheep and its significance for productivity. Animal Production, v. 50, p. 99-109, 1990.

ALBERS, G. A. A. et al. The genetics of resistance and resilience to Haemonchus contortus infection in young Merino sheep. International Journal for Parasitology, v. 17, n. 7, p. 1355-1363, 1987.

AMARANTE, A. F. T. et al. Efeito da administração de oxfendazol, ivermectina e levamisol sobre os exames coproparasitológicos de ovinos. Braz. J. Vet. Res. Anim. Sci., v. 29, p. 31-38, 1992.

AMARANTE, A. F. T. Resistência genética a helmintos gastrintestinais. In: V SIMPÓSIO DA SOCIEDADE BRASILEIRA DE MELHORAMENTO ANIMAL, 4., 2004, Pirassununga. Anais... Sociedade Brasileira de Melhoramento Animal, Pirassununga, 2004. 1 CD-ROM.

BATH, G. F. et al. Sustainable approaches for managing haemonchosis in sheep and goats. Rome: FAO, 2001. 114 p.

BISSET, S. A. et al. Phenotypic and genetic relationships amongst FAMACHA $^{\odot}$ score, faecal egg count and performance data in Merino Sheep exposed to Haemonchus contortus infection in South Africa. In: INTERNATIONAL SHEEP VETERINARY CONGRESS, 57., 2001, Stellenbosch. Proceedings...Stellenbosch, South Africa, Jan. 2001. 
BRICARELLO, P. A. et al. Worm burden and immunological responses in Corriedale and Crioula Lanada sheep following natural infection with Haemonchus contortus. Small Rumin. Res., v. 51, p. 75-83, 2004.

BUDDLE, B. M. et al. Association of blood eosinophilia with the expression of resistance in Romney lambs to nematodes. International Journal for Parasitology, v. 22, n. 7, p. 955-960, 1992.

COLES, G. C. et al. The detection of anthelmintic resistance in nematodes of veterinary importance. Veterinary Parasitology, v. 136, p. 167-185, 2006.

DORCHIES, P. Reduced egg counts in mixed infections with Oestrus ovis and Haemonchus contortus : influence of eosinophils? Vet. Res., v. 83, p. 727-730, 1997.

GILL, H. S. Genetic control of acquired resistance to haemonchosis in Merino lambs. Parasite Immunology, v. 13, p. 617-628, 1991.

GOOD, B. et al. Texel sheep are more resistant to natural nematode challenge than Suffolk sheep based on fecal egg count and nematode burden. Veterinary Parasitology, v. 136, p. 317-327, 2006.

GORDON, H. McL.; WHITLOCK, H. V. A new technique for counting nematode eggs in sheep faeces. Journal of the Council for Scientific and Industrial Research, v. 12, p. 50, 1939.

JAIN, N. C. Schalm’s veterinary hematology. 4. ed. Philadelphia: Lea \& Febiger, 1986. 1221 p.

KASSAI, T. et al. Is there a relationship between haemoglobin genotype and the innate resistance to experimental Haemonchus contortus infection in Merino lambs? Veterinary Parasitology, v. 37, p. 61-77, 1990.

LI, Y.; MILLER, J. E.; FRANKE, D. E. Epidemiological observations and heterosis analysis of gastrointestinal nematode parasitism in Suffolk, Gulf Coast Native, and crossbred lambs. Vet. Parasitol., v. 98, 273-283, 2001.

LIMA, A. O. et al. Métodos de laboratório aplicados à clínica: técnica e interpretação. 6. ed. Rio de Janeiro: Guanabara Koogan, 1985.

Mac FARLANE, J. C. W.; CECIL, G. W. Eosinophil counting: a modification of pilots method. Brit. Med. J., v. 2, p. 1187, 1951.

NARI, A.; EDDI, C. Alternativas para el control de las verminosis en pequeños rumiantes. In: CONGRESSO BRASILEIRO DE ESPECIALIDADES EM MEDICINA VETERINÁRIA, 1., 2002, Curitiba, PR. Anais...Curitiba, PR, 2002. p. 86-89.

RAMOS, C. I. et al. Resistência de parasitos gastrintestinais de ovinos a alguns anti-helmínticos no Eestado de Santa Catarina, Brasil. Ciência Rural, v. 32, n. 3, p. 473-477, 2002.

ROBERTS, F. H. S.; O’SULLIVAN, P. J. Methods for egg counts and larval cultures for strongyles infecting the gastro-intestinal tract of cattle. Australian Journal of Agriculture Research, v. 1, p. 99, 1950.

SANGSTER, N. C. Anthelmintic resistance: past, present and future. Int. J. Parasitol., v. 29, p. 115124, 1999.

SOCCOL, V. T.; et al. Occurence of resistance to anthelmintics in sheep in Paraná State, Brazil. Veterinary Record, v. 139, p. 421-422, 1996.

SOTOMAIOR, C. S. Estudo de caracteres que possam auxiliar na identificação de ovinos resistentes e susceptíveis aos helmintos gastrintestinais. Curitiba, 1997. Dissertação (Mestrado em Ciências Veterinárias) - Setor de Ciências Agrárias, Universidade Federal do Paraná, Curitiba, 1997.

SOTOMAIOR, C. S.; CALDAS, J. S. Validação do método FAMACHA ${ }^{\odot}$ para caprinos. In: SEMINÁRIO DE INICIAÇÃO CIENTÍFICA, XII., e MOSTRA DE PESQUISA DA PUCPR, 6., 2004, Curitiba, PR. Resumos... Curitiba, PR: [s.n.], 2004. 78 p. 
SOTOMAIOR, C. S.; SOCCOL, V. T. Infecção parasitária em ovinos criados em sistema intensivo: Acompanhamento de evolução do parasitismo durante um ano. A Hora Veterinária, v. 119, p. 10-15, 2001.

SOTOMAIOR, C.; MILCZEWSKI, V; SCHWARTZ, M. G. Teste de anti-helmínticos em rebanhos caprinos do Estado do Paraná. In: CONGRESSO BRASILEIRO DE ESPECIALIDADES EM MEDiCINA VETERINÁRIA, I., 2002, Curitiba, PR. Anais... Curitiba, PR: [s.n.], 2002. 189 p.

SRÉTER, T.; KASSAI, T.; TAKÁCS, E. The heritability and specificity of responsiveness to infection with Haemonchus contortus in sheep. International Journal for Parasitology, v. 24, n. 6, p. 871-876, 1994.

STEAR, M. J.; et al. The repeatability of faecal egg counts, peripheral eosinophil counts, and plasma pepsinogen concentrations during deliberate infections with Ostertagia circumcincta. International Journal for Parasitology, v. 25, n. 3, p. 375-380, 1995.

THOMAZ-SOCCOL, V. et al. Resistance of gastrointestinal nematodes to anthelmintics in sheep (Ovis aries). Brazilian Archives of Biology and Technology, v. 47, n. 1, p. 41-47, 2004.

VAN WYK, J. A. The FAMACHA ${ }^{\odot}$ system in dynamic management of haemonchosis in small ruminants. In: CONGRESSO BRASILEIRO DE ESPECIALIDADES EM MEDICINA VETERINÁRIA CONBREMEV, I. 2002. Curitiba. Anais... Curitiba: SERZEGRAF, 2002, p. 85.

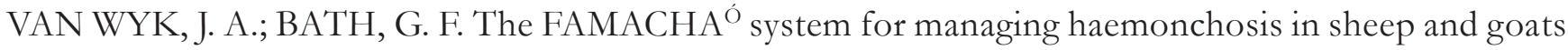
by clinically identifying individual animals for treatment. Vet. Res., v. 33, p. 509-529, 2002.

VIEIRA, L. V.; CAVALCANTE, C. R. Resistência anti-helmíntica em rebanhos caprinos no Estado do Ceará. Pesq. Vet. Bras., v. 19 (3/4), p. 99-103, jul./dez., 1999.

WALLER, P. J. Anthelmintic resistance. Vet. Parasitol., v. 72. p. 391-412, 1997.

WINDON, R. G.; DINEEN, J.K.; KELLY, J.D. The segregation of lambs into "responders" and "nonresponders": response to vaccination with irradiated Trichostrongylus colubriformis larvae before weaning. International Journal for Parasitology, v. 10, n. 1, p. 65-73, 1980.

WOOLASTON, R. R. Selection of Merino sheep for increased and decreased resistance to Haemonchus contortus: peri-parturient effects on faecal egg counts. International Journal for Parasitology, v. 22, n. 7, p. 947-953, 1992.

WOOLASTON, R.R.; BARGER, I.A.; PIPER, L.R. Response to helminth infection of sheep selected for resistance to Haemonchus contortus. International Journal for Parasitology, v. 20, n. 8, p. 1015-1018, 1990.

Recebido em: 28/02/2007

Received in: 02/28/2007

Aprovado em: 31/07/2007

Approved in: 07/31/2007 